\title{
Lower serum brain-derived neurotrophic factor levels are associated with failure to achieve remission in patients with major depression after escitalopram treatment
}

\author{
This article was published in the following Dove Press journal: \\ Neuropsychiatric Disease and Treatment \\ 23 July 2014 \\ Number of times this article has been viewed
}

\section{Bun-Hee Lee' \\ Young-Min Park ${ }^{2}$ \\ Tae Hyun Um ${ }^{3}$ \\ Sollip $\mathrm{Kim}^{3}$}

'Department of Psychiatry, Gangnam Eulji Hospital, Eulji University, Seoul, ${ }^{2}$ Department of Psychiatry, ${ }^{3}$ Department of Laboratory Medicine, Ilsan Paik Hospital, Inje University College of Medicine, Goyang, South Korea
Correspondence: Young-Min Park Department of Psychiatry, Ilsan Paik Hospital, Inje University College of Medicine, 2240 Daehwa-Dong, Ilsansu-ku, Goyang, Gyeonggi-do 4I I-706, South Korea Tel +82 3I 9l0 7260

Fax +82319107268

Email medipark@hanmail.net
Background: Remission, the primary goal of treatment for major depressive disorder (MDD), is the absence of significant signs or symptoms and the return to a state of normal functioning. A recent study found that the level of brain-derived neurotrophic factor (BDNF) increased after antidepressant treatment in remitted patients. This study evaluated serum BDNF levels in MDD patients with chronic maintenance treatment, and compared these between remission and nonremission groups.

Materials and methods: Serum BDNF levels were measured in 34 MDD patients and 35 healthy controls. The severity of depression was measured using the Hamilton Depression Rating Scale (Ham-D). The MDD patients were divided into remission and nonremission groups according to a cutoff total Ham-D score of either $\leq 7$ or $\leq 6$.

Results: Serum BDNF levels differed significantly between the remission, nonremission, and healthy control groups $(P<0.05)$. The Bonferroni post hoc test confirmed that serum BDNF levels were significantly lower in the nonremission group than in the healthy-control group $(P<0.05)$, but did not differ significantly between the remission and healthy-control groups.

Limitations: This study included a small sample, and measured serum BDNF levels in the MDD patients at only one point during the maintenance treatment.

Conclusion: This study found that serum BDNF levels during maintenance treatment were lower in MDD patients with failure to achieve remission than in controls, while the remitted subjects had normalized serum BDNF levels. A lower level of serum BDNF during maintenance treatment is associated with failure to achieve remission in patients with major depression. Moreover, serum BDNF levels after chronic antidepressant treatment can be used as a biological marker for detecting nonremission.

Keywords: brain-derived neurotrophic factor, major depression, remission

\section{Introduction}

Remission is the primary goal of treatment for major depressive disorder (MDD). One study that followed MDD patients for 2 years found that the rate of relapse in MDD was higher in partial remission than in complete remission. ${ }^{1}$ The presence of residual symptoms even after remission is associated with an increased likelihood of relapse. ${ }^{2}$ Therefore, achieving remission in MDD treatment is clinically significant.

Remission is defined in the Diagnostic and Statistical Manual of Mental Disorders (DSM)-IV as the absence of significant signs or symptoms. Another concept of remission is a return to a state of normal functioning and minimal symptoms. ${ }^{3}$ Several clinical 
studies have attempted to define cutoff scores for remission based on depression-rating scales, including the Hamilton Depression Rating Scale (Ham-D) and the Montgomery Asberg Depression Rating Scale (MADRS). Remission has been defined as a total Ham-D-17 score of $\leq 7$ or $\leq 6$ after treatment. ${ }^{4,5}$ However, there is as yet no biological marker to predict or reflect a state of remission in MDD.

Brain-derived neurotrophic factor (BDNF) is an important neurotrophic factor. Neurotrophic factors, including BDNF, can play a critical role in the neurogenesis and neural plasticity of the adult brain. ${ }^{6}$ Furthermore, many animal and clinical studies have examined the role of BDNF in MDD, and it has been hypothesized that dysfunction of the neuronal networks is associated with the development of MDD, and that a gradual recovery of networks through neural plasticity can induce a response to antidepressants and improvement of MDD. ${ }^{7}$ Meta-analyses of clinical studies indicate that serum and plasma BDNF levels are decreased in MDD patients before treatment, and that these BDNF levels increase following a course of antidepressant treatment. ${ }^{8,9}$ Moreover, in some clinical studies, these increases in serum and plasma BDNF levels were found after 6 or 8 weeks of treatment in responders, but not in nonresponders to antidepressants. ${ }^{10,11}$ One pilot study involving $41 \mathrm{MDD}$ patients showed that absences of an increase in serum BDNF and an improvement in Ham-D scores from baseline to day 7 or 14 predicted later nonresponse and nonremission with moderate-to-high specificity. ${ }^{12}$ This suggests that serum BDNF can be used as a biological marker for predicting treatment failure in MDD patients. ${ }^{12}$ Therefore, serum BDNF levels measured during maintenance treatment with antidepressants were hypothesized to remain low in MDD subjects who fail to achieve remission, and serum BDNF levels after chronic antidepressant treatment can be used as a biological marker for detecting nonremission. The present study aimed to determine whether a low serum BDNF level after chronic antidepressant medication is associated with a failure to achieve remission in MDD patients. This was achieved by measuring serum BDNF levels in healthy subjects and in MDD patients with and without remission.

\section{Materials and methods Subjects}

This study focused on 34 MDD consecutive outpatients being treated with escitalopram. The patients (ten males and 24 females, age $47.0 \pm 18.4$ years [mean \pm standard deviation]) were diagnosed with MDD according to the DSM-IV. Patients with any past or current history of Axis I or II psychiatric disorders other than MDD or with a personal history of major medical and/or neurological disorders were excluded. The following inclusion criteria were applied to enroll MDD patients in this study: 1) being in the first episode of MDD or having the first experience of antidepressant treatment, or no history of antidepressant treatment within 12 weeks before first visiting our hospital; 2) having a total Beck Depression Inventory (BDI) score exceeding 19 prior to the treatment; 3) having received escitalopram monotherapy for at least 3 months; and 4) having received the same dose of escitalopram per day for more than 2 months as maintenance treatment. The BDI score was $28.36 \pm 6.58$ before the treatment, the duration of total treatment for the present episode was $7.7 \pm 5.0$ months, and the duration of the maintenance phase was $5.29 \pm 4.87$ months. The included patients had also been taking benzodiazepines, including alprazolam or clonazepam.

Thirty-five healthy controls (12 males and 23 females, age 40.8 \pm 9.4 years) comprising randomly selected healthy volunteers also participated in this study. The controls had no personal or familial psychiatric or medical history. All subjects had been free of chronic and acute physical illnesses for at least 4 weeks prior to enrollment. The controls had normal laboratory findings for blood chemistry, renal function, thyroid function, liver function, and electrocardiography. The sex and age distributions did not differ significantly between the MDD patients and healthy controls $\left(\chi^{2}=0.189, P=0.664\right.$, and $t=1.749, P=0.087$; respectively).

The study protocol was approved by the institutional review board of Inje University. Written informed consent to participate was obtained from all subjects before beginning the investigation.

\section{Assessment}

\section{Clinical evaluation}

Demographic and clinical data were obtained from the subjects through interview. The severity of current depression was assessed by a trained psychiatrist using the Ham-D and BDI at the same time point. Remission of MDD patients was defined in two ways: as a total Ham-D score of $\leq 7$ or $\leq 6$. The MDD patients were divided into those with remission (remission group) and those without remission (nonremission group). The total Ham-D score and the scores on the Ham-D anxiety, depression, insomnia, and somatic symptom subscales were determined. ${ }^{13}$

\section{Serum BDNF measurement}

Blood samples were taken to measure BDNF levels at a single time point, and were centrifuged to separate the serum. Serum BDNF levels were analyzed using enzyme-linked 
immunosorbent assay (ELISA) kits (Human Quantikine ${ }^{\circledR}$ BDNF; R\&D Systems, Minneapolis, MN, USA). Each assay was performed in duplicate. The actual concentration for each sample was calculated using the four-parameter-fit logistic curve equation. The ELISA plate readings were conducted using a microplate reader (VersaMax ${ }^{\mathrm{TM}}$; Molecular Devices, Sunnyvale, CA, USA).

\section{Statistical analysis}

Study groups were compared using the two-tailed $t$-test and analysis of variance for continuous variables, and using a $\chi^{2}$ test for discrete variables. Pearson's correlation coefficients were calculated to examine the relationships between serum BDNF and the clinical variables. A general linear model was used while controlling for covariates. The null hypothesis was rejected at $P<0.05$. The statistical analysis was conducted using the SAS 9.3 (SAS Institute, Cary, NC, USA) and SALT 2.5 (Melbourne, Australia) software packages.

\section{Results}

Serum BDNF levels were $21.20 \pm 7.21 \mathrm{ng} / \mathrm{mL}$ in the $34 \mathrm{MDD}$ patients and $24.89 \pm 7.38 \mathrm{ng} / \mathrm{mL}$ in the 35 healthy controls ( $t=-2.099, P=0.040)$. Based on a total Ham-D score of $\leq 7$ for remission, BDNF levels were $22.65 \pm 8.06 \mathrm{ng} / \mathrm{mL}$ in the remission group and $18.54 \pm 4.46 \mathrm{ng} / \mathrm{mL}$ in the nonremission group ( $t=1.627, P=0.114$ ). Serum BDNF levels differed significantly among the remission, nonremission, and healthy control groups $(F=3.511, d f=2,66 ; P=0.036)$. The Bonferroni post hoc test confirmed the presence of a significant difference in the serum BDNF levels between the nonremission and healthy control groups $(P=0.032)$, but not between the remission and healthy control groups $(P=0.775)$. Controlling for sex and age as covariates revealed that these two factors did not significantly affect the results $(P>0.05)$.

On the other definition of remission, of a total Ham-D score of $\leq 6$, BDNF levels were $22.92 \pm 8.16 \mathrm{ng} / \mathrm{mL}$ in the remission group and $18.42 \pm 4.29 \mathrm{ng} / \mathrm{mL}$ in the nonremission group $(t=1.834, P=0.043)$. Serum BDNF levels also differed significantly among the remission, nonremission, and healthy control groups using this definition of remission $(F=3.855, d f=2$, $66 ; P=0.026)$. The Bonferroni post hoc test showed that serum BDNF levels were significantly lower in the nonremission group than in the healthy control group $(P=0.022$, Figure 1$)$, whereas they did not differ significantly between the remission and healthy control groups $(P=0.976)$. Controlling for sex and age as covariates revealed that these two factors did not significantly affect the results $(P>0.05)$.

Before the treatment, the BDI scores in MDD patients did not differ significantly between the remission and nonremission groups $(P>0.05$, Table 1$)$. After the treatment,

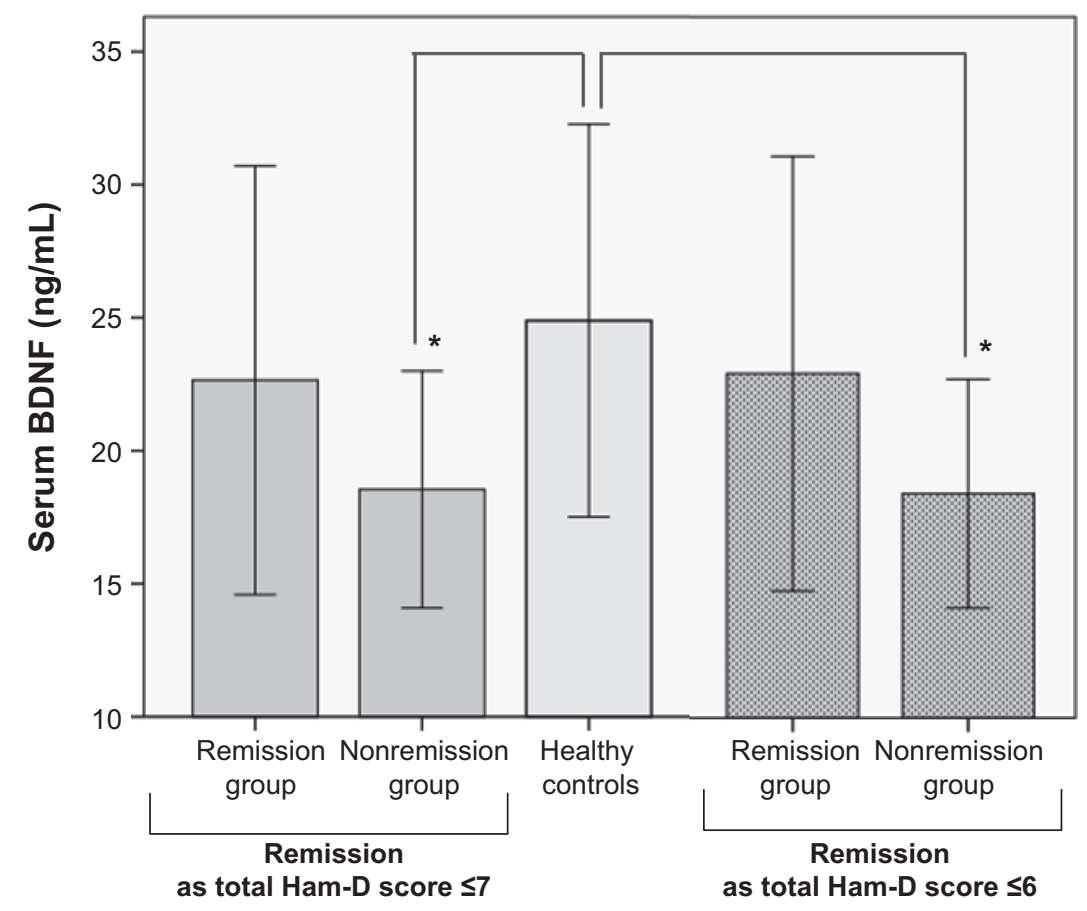

Figure I Difference in serum BDNF among the remission and nonremission groups and healthy controls.

Notes: Data are presented as means \pm standard deviation. Differences from healthy controls are indicated $(* P<0.05)$, as analyzed by analysis of variance. Serum BDNF levels of MDD patients with remission during the maintenance phase of treatment were comparable to those of healthy controls. MDD patients without remission had significantly lower serum BDNF than healthy controls.

Abbreviations: BDNF, brain-derived neurotrophic factor; Ham-D, Hamilton Depression Rating Scale; MDD, major depressive disorder. 
Table I Comparison of demographic and clinical variables and serum BDNF levels between groups with and without remission, defined according to the score for total Ham-D score

\begin{tabular}{|c|c|c|c|c|c|c|}
\hline \multirow[t]{2}{*}{ Variable } & \multicolumn{3}{|c|}{ Remission defined as $\leq \mathbf{7}$ for total Ham-D score } & \multicolumn{3}{|c|}{ Remission defined as $\leq 6$ for total Ham-D score } \\
\hline & $\begin{array}{l}\text { Remission group } \\
(n=22)\end{array}$ & $\begin{array}{l}\text { Nonremission group } \\
(\mathrm{n}=\mid 2)\end{array}$ & $P$ & $\begin{array}{l}\text { Remission group } \\
(n=2 I)\end{array}$ & $\begin{array}{l}\text { Nonremission group } \\
(n=\mid 3)\end{array}$ & $P$ \\
\hline Age, years & $47.5 \pm 17.3$ & $46.0 \pm 21.0$ & 0.824 & $48.8 \pm 16.6$ & $44.0 \pm 21.4$ & 0.467 \\
\hline Sex (male/female) & $9 / 13$ & $\mathrm{I} / \mathrm{II}$ & $0.046^{*}$ & $8 / 13$ & $2 / 11$ & 0.158 \\
\hline Escitalopram dose, $\mathrm{mg}$ & $11.8 \pm 5.0$ & $11.2 \pm 4.3$ & 0.743 & $11.7 \pm 5.1$ & $11.5 \pm 4.3$ & 0.940 \\
\hline \multicolumn{7}{|l|}{ Total BDI score } \\
\hline Before treatment & $26.67 \pm 6.68$ & $29.63 \pm 6.65$ & 0.427 & $28.00 \pm 6.52$ & $28.56 \pm 7.00$ & 0.887 \\
\hline After treatment & $5.91 \pm 6.98$ & $21.75 \pm 9.55$ & $<0.0 I^{* *}$ & $5.38 \pm 5.57$ & $21.38 \pm 9.24$ & $<0.0 I^{* * *}$ \\
\hline $\begin{array}{l}\text { Total Ham-D score } \\
\text { after treatment }\end{array}$ & $3.32 \pm 2.30$ & $12.67 \pm 3.89$ & $<0.0 \mathrm{I}^{* *}$ & $3.14 \pm 2.20$ & $12.23 \pm 4.04$ & $<0.01 * *$ \\
\hline Depression subscale & $0.77 \pm 0.97$ & $4.58 \pm 1.62$ & $<0.0 I^{* *}$ & $0.7 I \pm 0.96$ & $4.38 \pm 1.71$ & $<0.01 * *$ \\
\hline Anxiety subscale & $0.95 \pm 0.84$ & $3.58 \pm 2.19$ & $0.002 * *$ & $0.90 \pm 0.83$ & $3.46 \pm 2.15$ & $0.001 * *$ \\
\hline Insomnia subscale & $0.82 \pm 1.18$ & $2.25 \pm 1.60$ & $0.006 * *$ & $0.8 I \pm I .21$ & $2.15 \pm 1.57$ & $0.008 * *$ \\
\hline Somatic subscale & $0.77 \pm 0.97$ & $2.25 \pm|.7|$ & $0.015^{*}$ & $0.7 I \pm 0.96$ & $2.23 \pm 1.64$ & $0.008 * *$ \\
\hline BDNF, ng/mL & $22.65 \pm 8.06$ & $18.54 \pm 4.46$ & 0.114 & $22.92 \pm 8.16$ & $18.42 \pm 4.29$ & $0.043^{*}$ \\
\hline
\end{tabular}

Notes: $* P<0.05 ; * * P<0.01$. Except where stated otherwise, data are means \pm standard deviation.

Abbreviations: BDNF, brain-derived neurotrophic factor; Ham-D, Hamilton Depression Rating Scale, BDI, Beck Depression Inventory.

the total Ham-D score, the scores on the Ham-D subscales, and the total BDI score differed significantly between the remission and nonremission groups (Table 1), whereas age and escitalopram dose did not.

The total Ham-D scores were significantly and negatively correlated with BDNF levels in all MDD patients $(r=-0.37$, $P=0.031$ ). The score on the Ham-D anxiety subscale also significantly negatively correlated with BDNF $(r=-0.34$, $P=0.049)$. However, BDNF levels were not significantly correlated with scores on the Ham-D depression, insomnia, and somatic symptoms subscales, or with the total BDI score (data not shown). There was no significant correlation between BDNF and escitalopram dose or duration of maintenance treatment $(r=-0.249, P=0.156 ; r=0.250, P=0.156$; respectively).

\section{Discussion}

The present study found that serum BDNF levels during the maintenance phase of treatment were significantly lower in MDD patients with failure to achieve remission than in healthy controls, and were comparable in MDD patients in remission and healthy controls. These results were observed using both cutoff scores for remission (ie, total Ham-D scores of $\leq 7$ and $\leq 6$ ).

Many clinical studies and meta-analyses suggest that serum BDNF levels in MDD patients increase after antidepressant treatment. ${ }^{8,10,14,15}$ One clinical study of 42 MDD patients observed a significant increase in serum BDNF from baseline to after 8 weeks of treatment in patients who responded to treatment, but not in nonresponders. ${ }^{10}$
Moreover, serum BDNF levels after 8 weeks of treatment differed significantly between responders and nonresponders. A recent study examined serum BDNF levels in 962 current MDD patients, 700 subjects who had been fully remitted for more than 6 months, and 382 healthy controls. ${ }^{16}$ It found that the serum BDNF level was significantly lower in current MDD patients and was normalized in the remitted subjects. A naturalistic study found that plasma BDNF levels in 79 moderate-to-severe MDD patients increased significantly with clinical improvement in the remission group (MADRS score of $\leq 8$ after 12 weeks of treatment). ${ }^{17}$ However, the nonresponders had significantly decreased BDNF levels after the treatment. These reports are consistent with the present findings that serum BDNF levels during maintenance treatment did not differ between patients in remission and healthy controls. However, MDD patients who failed to achieve remission had significantly lower serum BDNF levels even after treatment. In contrast, other studies have found low levels of serum BDNF among MDD patients in a euthymic state (Ham-D score of $<8$ for at least 1 month) or in early remission (1-6 months). ${ }^{16,18}$ This discrepancy could be explained by the assumption that serum BDNF levels can remain low when clinical improvement has been established in early remission, ${ }^{16}$ ie, the normalization of serum BDNF might require a sufficient duration of clinical improvement or remission. In addition, this inconsistency indicates that the criteria used for remission based on depression-rating scales (including Ham-D) are not sufficient to define remission in MDD. Together, these results indicate that a lower serum BDNF level after chronic treatment might be associated 
with a failure to achieve remission, and serum BDNF levels after chronic antidepression treatment can be used as a biological marker for detecting nonremission. Moreover, we should consider not only clinical factors, such as a cutoff total Ham-D score of either $\leq 7$ or $\leq 6$, but also biological factors, such as serum BDNF levels simultaneously in order to detect remission in MDD patients.

The present study also found significant negative correlations between serum BDNF levels, total Ham-D scores, and scores on the Ham-D anxiety subscale among the MDD patients during the maintenance treatment. Clinical improvement might lead to an increase in serum BDNF during treatment. However, findings regarding the relationship between serum BDNF levels and the severity of depression have been inconsistent: some studies have found a negative correlation between these two parameters, ${ }^{14,19}$ while others have not. ${ }^{16,20,21}$ In addition, scores on the Ham-D depression, anxiety, insomnia, and somatic symptom subscales differed significantly between the remission and nonremission groups in the present study. However, the score on the Ham-D anxiety subscale was significantly negatively correlated with serum BDNF levels, which indicates that residual anxiety symptoms might be associated with the presence of low levels of serum BDNF in MDD patients who will not achieve remission.

This study was subject to several limitations. First, the serum BDNF levels were measured in MDD patients only once during the maintenance treatment. Therefore, it was not possible to observe changes in serum BDNF levels between the period before and after treatment with antidepressants. Second, this study had a relatively small sample. A recent meta-analysis addressed the issue of a small sample or underpowered design in clinical studies, and suggested that a sample comprising at least 57 subjects in each group is necessary to detect differences in serum BDNF concentrations between healthy controls and depressed subjects reliably. ${ }^{22}$ Despite these limitations, the present study found that serum BDNF levels during the maintenance treatment were lower in MDD patients who failed to achieve remission. Also, defining and detecting remission in MDD appears to require the use of several indicators simultaneously, including a clinical factor, such as the Ham-D score, and a biological factor, such as the serum BDNF level. Further studies involving larger samples are needed to replicate and confirm these findings.

\section{Acknowledgments}

This study was supported by a grant from National Research Foundation of Korea, funded by Ministry of Education and
Science Technology (NRF2011-0010562). The authors thank Jo Bonu for her assistance with data collection.

\section{Disclosure}

The authors report no conflicts of interest in this work.

\section{References}

1. Pintor L, Gastó C, Navarro V, Torres X, Fañanas L. Relapse of major depression after complete and partial remission during a 2-year follow-up. $J$ Affect Disord. 2003;73(3):237-244.

2. Nierenberg AA, Husain MM, Trivedi MH, et al. Residual symptoms after remission of major depressive disorder with citalopram and risk of relapse: a STAR*D report. Psychol Med. 2010;40(1):41-50.

3. Frank E, Prien RF, Jarrett RB, et al. Conceptualization and rationale for consensus definitions of terms in major depressive disorder. Remission, recovery, relapse, and recurrence. Arch Gen Psychiatry. 1991;48(9): 851-855.

4. Nierenberg AA, DeCecco LM. Definitions of antidepressant treatment response, remission, nonresponse, partial response, and other relevant outcomes: a focus on treatment-resistant depression. J Clin Psychiatry. 2001;62 Suppl 16:5-9.

5. Riedel M, Möller HJ, Obermeier M, et al. Response and remission criteria in major depression - a validation of current practice. J Psychiatr Res. 2010;44(15):1063-1068.

6. Warner-Schmidt JL, Duman RS. Hippocampal neurogenesis: opposing effects of stress and antidepressant treatment. Hippocampus. 2006; 6(3): 239-249.

7. Castrén E, Võikar V, Rantamäki T. Role of neurotrophic factors in depression. Curr Opin Pharmacol. 2007;7(1):18-21.

8. Sen S, Duman R, Sanacora G. Serum brain-derived neurotrophic factor, depression, and antidepressant medications: meta-analyses and implications. Biol Psychiatry. 2008;64(6):527-532.

9. Bocchio-Chiavetto L, Bagnardi V, Zanardini R, et al. Serum and plasma BDNF levels in major depression: a replication study and meta-analyses. World J Biol Psychiatry. 2010;11(6):763-773.

10. Yoshimura R, Mitoma M, Sugita A, et al. Effects of paroxetine or milnacipran on serum brain-derived neurotrophic factor in depressed patients. Prog Neuropsychopharmacol Biol Psychiatry. 2007;31(5): 1034-1037.

11. Lee HY, Kim YK. Plasma brain-derived neurotrophic factor as a peripheral marker for the action mechanism of antidepressants. Neuropsychobiology. 2008;57(4):194-199.

12. Tadić A, Wagner S, Schlicht KF, et al. The early non-increase of serum BDNF predicts failure of antidepressant treatment in patients with major depression: a pilot study. Prog Neuropsychopharmacol Biol Psychiatry. 2011;35(2):415-420.

13. Shafer AB. Meta-analysis of the factor structures of four depression questionnaires: Beck, CES-D, Hamilton, and Zung. J Clin Psychol. 2006; 62(1):123-146.

14. Gonul AS, Akdeniz F, Taneli F, Donat O, Eker C, Vahip S. Effect of treatment on serum brain-derived neurotrophic factor levels in depressed patients. Eur Arch Psychiatry Clin Neurosci. 2005;255(6): 381-386.

15. Brunoni AR, Lopes M, Fregni F. A systematic review and meta-analysis of clinical studies on major depression and BDNF levels: implications for the role of neuroplasticity in depression. Int $J$ Neuropsychopharmacol. 2008;11(8):1169-1180.

16. Molendijk ML, Bus BA, Spinhoven P, et al. Serum levels of brainderived neurotrophic factor in major depressive disorder: state-trait issues, clinical features and pharmacological treatment. Mol Psychiatry. 2011;16(11):1088-1095.

17. Kurita M, Nishino S, Kato M, Numata Y, Sato T. Plasma brain-derived neurotrophic factor levels predict the clinical outcome of depression treatment in a naturalistic study. PLoS One. 2012;7(6):e39212. 
18. Monteleone P, Serritella C, Martiadis V, Maj M. Decreased levels of serum brain-derived neurotrophic factor in both depressed and euthymic patients with unipolar depression and in euthymic patients with bipolar I and II disorders. Bipolar Disord. 2008;10(1):95-100.

19. Dell'Osso L, Del Debbio A, Veltri A, et al. Associations between brainderived neurotrophic factor plasma levels and severity of the illness, recurrence and symptoms in depressed patients. Neuropsychobiology. 2010;62(4):207-212.

20. Lee BH, Kim H, Park SH, Kim YK. Decreased plasma BDNF level in depressive patients. J Affect Disord. 2007;101(1-3):239-244.
21. Jevtović S1, Karlović D, Mihaljević-Peleš A, Šerić V, Vrkić N, Jakšić N. Serum brain-derived neurotrophic factor (BDNF): the severity and symptomatic dimensions of depression. Psychiatr Danub. 2011;23(4): 363-369.

22. Molendijk ML, Spinhoven P, Polak M, Bus BA, Penninx BW, Elzinga BM. Serum BDNF concentrations as peripheral manifestations of depression: evidence from a systematic review and meta-analyses on 179 associations (N=9484). Mol Psychiatry. Epub 2013 Aug 20.

\section{Publish your work in this journal}

Neuropsychiatric Disease and Treatment is an international, peerreviewed journal of clinical therapeutics and pharmacology focusing on concise rapid reporting of clinical or pre-clinical studies on a range of neuropsychiatric and neurological disorders. This journal is indexed on PubMed Central, the 'PsycINFO' database and CAS, and is the official journal of The International Neuropsychiatric Association (INA). The manuscript management system is completely online and includes a very quick and fair peer-review system, which is all easy to use. Visit http://www.dovepress.com/testimonials.php to read real quotes from published authors.

Submit your manuscript here: http://www.dovepress.com/neuropsychiatric-disease-and-treatment-journal 